\title{
An increased neutrophil-to-lymphocyte ratio predicts poorer survival following recurrence for patients with breast cancer
}

\author{
TOSHIAKI IWASE, TAKAFUMI SANGAI, MASAHIRO SAKAKIBARA, JUNTA SAKAKIBARA, EMI ISHIGAMI, \\ SHOUKO HAYAMA, AYAKO NAKAGAWA, TAKAHITO MASUDA, SHUNSUKE TABE and TAKESHI NAGASHIMA \\ Department of General Surgery, Chiba University Hospital, Chiba 2608677, Japan
}

Received July 26, 2016; Accepted September 12, 2016

DOI: $10.3892 / \mathrm{mco} .2016 .1101$

\begin{abstract}
The aim of the present study was to evaluate the association between changes in the neutrophil-to-lymphocyte ratio and the survival rate, as well as tumor subtype, in recurrent breast cancer. Patients with recurrent breast cancer following surgery were included in this study. NLR was calculated and compared between two time points: Pre-treatment and recurrence. The associations between the longitudinal NLR change, the NLR at the time of recurrence and overall survival following recurrence (OSrec) were evaluated. A total of 89 patients were evaluated. NLR increased by 0.59 at recurrence, as compared with the initial treatment $(\mathrm{P}<0.05)$. The triple negative (TN) type demonstrated 4.59 in NLR, which was the highest among the four subtypes at the time of recurrence $(\mathrm{P}<0.05)$. The highest change (an increase of 2.0) was observed in TN type cancer $(\mathrm{P}<0.05)$. Patients with high NLR upon recurrence demonstrated significantly shorter OSrec rates $(\mathrm{P}<0.05)$. On the other hand, patients with an NLR increased by more than a third quartile demonstrated a shorter OSrec rate $(\mathrm{P}=0.06)$. When adjusted by covariates, the NLR and tumor subtype were determined to be associated with OSrec $(\mathrm{P}<0.05)$. Therefore, an increased NLR predicts survival, even in patients with recurrent breast cancer, and the NLR is potentially useful as an inflammation marker for TN breast cancer.
\end{abstract}

\section{Introduction}

Breast cancer is the second most common cancer in the world, and the most frequent cancer in women, with 1.67 million newly diagnosed cases in 2012 (1). Furthermore, it is estimated that $20-30 \%$ of all breast cancer cases will become metastatic (2). Although survival outcomes for patients with

Correspondence to: Dr Toshiaki Iwase, Department of General Surgery, Chiba University Hospital, 1-8-1 Inohana Chuo-ku, Chiba 2608677, Japan

E-mail: tiwase@chiba-u.jp

Key words: neutrophil-to-lymphocyte ratio, triple negative breast cancer, overall survival after recurrence, breast cancer metastatic breast cancer have improved owing to modern chemotherapy developments, a method for predicting lifetime expectancy has not been established.

The neutrophil-to-lymphocyte ratio (NLR) has been widely used as an evaluation tool for inflammatory and immune responses. Since increased levels of inflammation, or of an immune reaction, reflect tumor aggressiveness or the host condition, NLR may also be used as a predictor for survival in patients with solid malignancies (3-5). A high value for the NLR is linked with cancer-associated inflammation. Neutrophilia resulting from inflammation inhibits the immune system by suppressing the cytolytic activity of immune cells, including lymphocytes, $\mathrm{T}$ cells and natural killer cells, thereby promoting cancer progression (6).

Several studies have demonstrated that NLR is useful in terms of predicting survival prognoses in breast cancer patients $(5,7,8)$. Cross-sectional studies revealed that patients with a higher NLR had poorer survival outcomes. However, the majority of studies targeted patients with early breast cancer, and no studies, thus far, have focused on metastatic breast cancer (5,7-9).

The aim of present study was to evaluate the usefulness of NLR as a prediction tool for patients with recurrent breast cancer.

\section{Patients and methods}

Inclusion criteria. A total of 89 patients who had breast cancer recurrence during the follow-up period after surgery between January 2005 and December 2014 at Chiba Graduate School of Medicine were enrolled in the present study. Ethical approval was obtained for this study, and informed consent was obtained from each of the patients prior to their inclusion in the present study. Recurrent sites included local and distant sites: The remnant breast, regional lymph nodes, bone, brain, and internal organs. Patients who already had a metastatic site at the time of initial diagnosis were excluded from the present study to avoid selection and timing biases.

Neutrophil-to-lymphocyte ratio. NLR calculations were made by dividing the serum neutrophil count by the lymphocyte count. According to previous reports, the NLR value may range widely, from 2.5 to 4.0 , and a standard cut-off value for a high NLR has not been established (6). A value of 3.0 was 
selected for the present study on the basis of a previous report that focused on the association between breast cancer subtypes and NLR (9). To compare differences in NLR from the time of initial treatment to recurrence, NLR was calculated at each of these time points. Specimens harvested within one month following chemotherapy were avoided, so as to eliminate any effects due to drugs on NLR.

Treatments for metastatic breast cancer. Tumor staging upon initial treatment was summarized using the American Joint Committee on Cancer/Union for International Cancer Control tumor-lymph node-metastasis (TNM) classification system (10). The current treatment algorism established by Hortobagyi (11), based on tumor subtype, was followed. Bone-modifying agents were added when patients presented with bone metastasis.

Covariates. The following variables were selected as covariates in multivariate analysis, as obtained from medical records: Menopausal status, body mass index (BMI; categorized by the World Health Organization), lactate dehydrogenase (LDH) and subtype.

Statistical analysis. Univariate analyses were performed to compare clinical and pathological characteristics between two groups categorized by the NLR value (NLR $<3$ vs. NLR $\geq 3$ ). Statistical tests were chosen on the basis of a variable distribution. Student's $t$-tests were used to compare normally distributed variables; Mann-Whitney U tests were used for non-normally distributed variables. One-way analysis of variance (ANOVA) and one-way factorial ANOVA were used to compare NLR values across tumor subtypes between initial treatment and recurrence. The relative NLR change from initial treatment to the time of recurrence was categorized into two groups according to the third quartile of NLR change (third quartile, 1.66).

Overall survival following recurrence (OSrec) was defined as the time between recurrence and death due to breast cancer. Survival curves were obtained using Kaplan-Meier survival analysis, and the curves were subsequently compared using a log-rank test. Multivariate analysis was also performed to identify factors that independently influenced survival. All tests were two-tailed, and $\mathrm{P}<0.05$ was considered to indicate a statistically significant difference. SPSS ${ }^{\circledR}$ software version 23 (IBM, Inc., Tokyo, Japan) was used for the statistical analysis, and GraphPad Prism $6{ }^{\circledR}$ (GraphPad Software, Inc., La Jolla, CA, USA) was used to generate the graphs.

\section{Results}

Demographic and clinical characteristics. A total of 73 patients $(82 \%)$ had tumors $\geq 2 \mathrm{~cm}$ in size, and 48 patients (54\%) had axillar metastasis. In examining the tumor subtypes based on immunohistochemical staining, estrogen receptor (ER)-positive/human epidermal growth factor 2 receptor (HER2)-negative (ER $\left.{ }^{+} / \mathrm{HER}^{-}\right), \mathrm{ER}^{+} / \mathrm{HER}^{+}, \mathrm{HER} 2$ type and triple negative (TN) type were present in 31 (35\%), 20 (22\%), $14(16 \%)$ and $24(27 \%)$ cases, respectively. A total of 83 patients (93\%) received chemotherapy [47 $(53 \%)$ neoadjuvant and $36(40 \%)$ adjuvant chemotherapy cases]. Thirty-eight cases
Table I. Demographics of the patients.

\begin{tabular}{|c|c|c|}
\hline Variable & $\begin{array}{l}\text { No. of patients } \\
\qquad(\mathrm{n}=89)\end{array}$ & $\%$ \\
\hline Age (year, average \pm SD) & $50.9 \pm 11.3$ & \\
\hline BMI $\left(\mathrm{kg} / \mathrm{m}^{2}\right.$, average $\left.\pm \mathrm{SD}\right)$ & $22.3 \pm 3.6$ & \\
\hline \multicolumn{3}{|l|}{ WHO BMI classification } \\
\hline Underweight (BMI <18.5 kg/m²) & 9 & 10 \\
\hline Normal range $\left(18.5 \leq \mathrm{BMI}<25 \mathrm{~kg} / \mathrm{m}^{2}\right)$ & 59 & 66 \\
\hline Overweight $\left(25 \leq \mathrm{BMI}<30 \mathrm{~kg} / \mathrm{m}^{2}\right)$ & 19 & 21 \\
\hline Obese $\left(\mathrm{BMI} \geq 30 \mathrm{~kg} / \mathrm{m}^{2}\right)$ & 2 & 3 \\
\hline \multicolumn{3}{|l|}{ Menopausal status } \\
\hline Premenopausal & 40 & 45 \\
\hline Postmenopausal & 49 & 55 \\
\hline \multicolumn{3}{|l|}{ T stage } \\
\hline 1 & 16 & 18 \\
\hline 2 & 53 & 60 \\
\hline 3 & 8 & 9 \\
\hline 4 & 12 & 13 \\
\hline \multicolumn{3}{|l|}{ N stage } \\
\hline 0 & 41 & 46 \\
\hline 1 & 36 & 40 \\
\hline 2 & 2 & 2 \\
\hline 3 & 10 & 12 \\
\hline \multicolumn{3}{|l|}{ Subtypes } \\
\hline $\mathrm{ER}^{+}, \mathrm{HER}^{-}$ & 31 & 35 \\
\hline $\mathrm{ER}^{+}, \mathrm{HER}^{+}$ & 20 & 22 \\
\hline HER2 type & 14 & 16 \\
\hline Triple negative type & 24 & 27 \\
\hline \multicolumn{3}{|l|}{ Neoadjuvant chemotherapy } \\
\hline Yes & 47 & 53 \\
\hline No & 42 & 47 \\
\hline \multicolumn{3}{|l|}{ Adjuvant chemotherapy } \\
\hline Yes & 36 & 40 \\
\hline No & 53 & 60 \\
\hline \multicolumn{3}{|l|}{ Recurrent site } \\
\hline Local & 21 & 24 \\
\hline Lymph node & 17 & 19 \\
\hline Lung & 15 & 17 \\
\hline Liver & 11 & 12 \\
\hline Bone & 12 & 13 \\
\hline Brain & 6 & 7 \\
\hline Other & 7 & 8 \\
\hline
\end{tabular}

SD, standard deviation; BMI, body mass index; WHO, World Health Organization; ER, estrogen receptor; HER, human epidermal growth factor 2 receptor.

(43\%) demonstrated local recurrence, and 51 (57\%) had distant metastasis (Table I).

Changes in NLR. The TN type demonstrated an NLR of 4.59, which was highest among the four tumor subtypes at the time 
Table II. Longitudinal change in the neutrophil-to-lymphocyte ratio from the time of initial treatment to recurrence of the cancer.

\begin{tabular}{|c|c|c|c|c|c|c|c|c|c|}
\hline \multirow[b]{2}{*}{ Variable } & \multicolumn{2}{|c|}{ Initial } & \multicolumn{2}{|c|}{ Recurrent } & \multicolumn{3}{|c|}{ Change } & \multicolumn{2}{|c|}{ Relative change (\%) } \\
\hline & Mean & $\mathrm{SD}$ & Mean & $\mathrm{SD}$ & Mean & $95 \% \mathrm{CI}$ & P-value & Mean & $95 \% \mathrm{CI}$ \\
\hline NLR & 2.24 & 1.01 & 2.83 & 2.18 & 0.59 & $0.15-1.04$ & $<0.05$ & 138.4 & $118.1-158.7$ \\
\hline $\mathrm{ER}^{+} / \mathrm{HER}^{-}$ & 2.00 & 0.92 & 2.67 & 2.17 & 0.66 & $-0.11-1.43$ & 0.09 & 154.5 & $122.8-186.1$ \\
\hline $\mathrm{ER}^{+} / \mathrm{HER}^{+}$ & 2.44 & 1.24 & 2.46 & 1.47 & 0.03 & $-0.63-0.68$ & 0.94 & 128.9 & $77.6-180.3$ \\
\hline TN type & 2.59 & 0.77 & 4.59 & 3.1 & 2.00 & $0.23-3.77$ & $<0.05$ & 92.1 & $56.9-127.2$ \\
\hline HER2 type & 2.17 & 1.00 & 2.33 & 1.57 & 0.16 & $-0.53-0.85$ & 0.63 & 152.6 & 106.6-198.6 \\
\hline
\end{tabular}

NLR, neutrophil-to-lymphocyte ratio; ER, estrogen receptor; HER, human epidermal growth factor receptor; TN, triple negative; SD, standard deviation; CI, confidence interval.

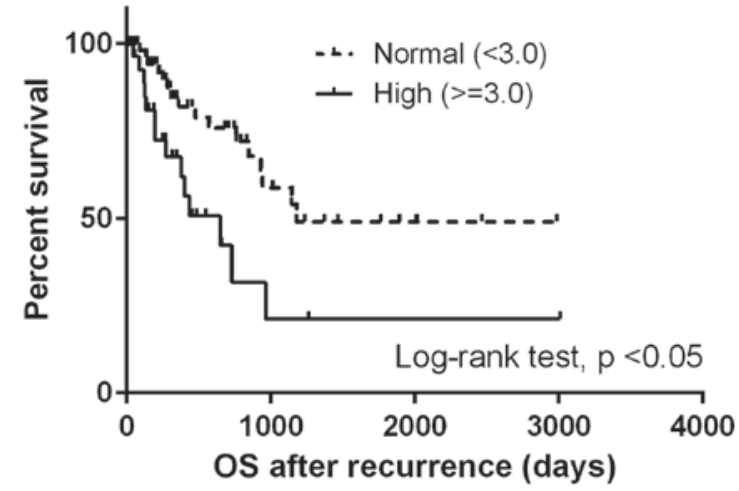

Figure 1. Comparison of survival curves for overall survival following recurrence stratified by the neutrophil-to-lymphocyte ratio at the time of recurrence. OS, overall survival.

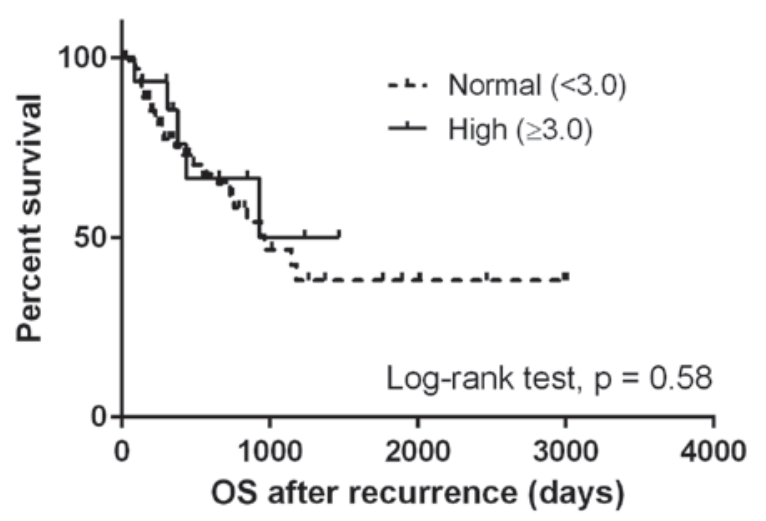

Figure 2. Comparison of survival curves for overall survival following recurrence stratified by the neutrophil-to-lymphocyte ratio at the time of initial treatment. OS, overall survival.

of recurrence $(\mathrm{P}<0.05)$. On the other hand, the initial NLR was not significantly different $(\mathrm{P}=0.58$; Table II).

Overall, NLR increased by 0.59 at the time of recurrence, as compared with the initial treatment [95\% confidence interval (CI), 0.15-1.04; $\mathrm{P}<0.05]$. The highest change in NLR was observed in the TN type, which increased by 2.0 (95\% CI, 0.23-3.77; $\mathrm{P}<0.05$; Table II). Other subtypes also demonstrated an increased NLR between the two time points, although these changes did not reach the level of significance (Table II).

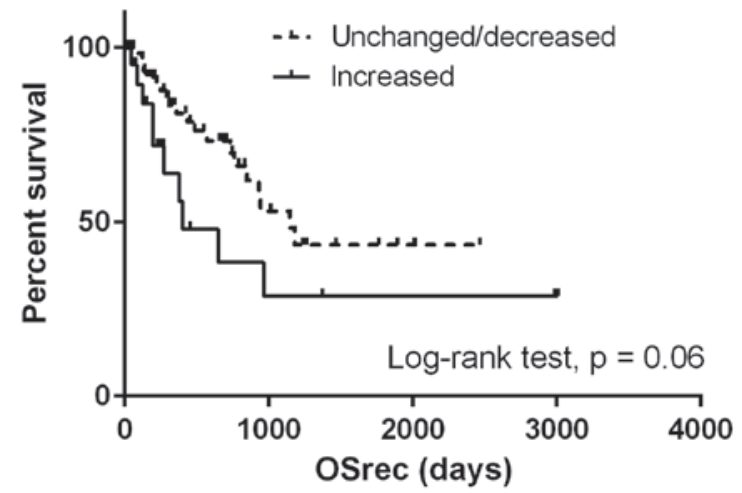

Figure 3. Comparison of survival curves for overall survival following recurrence stratified by longitudinal change in the neutrophil-to-lymphocyte ratio from the time of initial treatment to the recurrence. OSrec, overall survival following recurrence.

Survival analysis. The median OSrec was 964 days. Patients with a high NLR at the time of recurrence demonstrated significantly shorter OSrec times [Hazard ratio (HR), 2.68; 95\% CI, 1.29-5.57; P<0.05) (Fig. 1), whereas no difference was observed in the OSrec stratified by NLR at initial treatment ( $\mathrm{P}=0.58$; Fig. 2). Patients with NLR values increased by more than a third quartile demonstrated shorter OSrec times $(\mathrm{P}=0.06$; Fig. 3). The TN type demonstrated significantly shorter OSrec times among the four subtypes, with a median survival of 308 days $(\mathrm{P}<0.05)$.

When adjusted by covariates (menopausal status, BMI, LDH and subtypes), NLR and tumor subtype were significantly associated with OSrec (HR, 3.31; 95\% CI, 1.01-10.86 for the TN type, and HR, 2.93; 95\% CI, 1.29-6.64 for NLR>3.0; Table III).

\section{Discussion}

To the best of our knowledge, the present study is the first one to evaluate the predictive value of NLR changes in the recurrent breast cancer setting. It was determined that a higher NLR at the time of recurrence predicted poorer OSrec times in patients with breast cancer. Furthermore, TN breast cancer had significantly higher NLRs at the time of recurrence, as compared with other subtypes. 
Table III. The result of Cox proportional hazards model.

\begin{tabular}{|c|c|c|c|}
\hline Variable & $\begin{array}{c}\text { Hazard } \\
\text { ratio }\end{array}$ & $95 \% \mathrm{CI}$ & P-value \\
\hline NLR & & & $>0.05$ \\
\hline Normal $(<3.0)$ & Ref & & \\
\hline High $(\geq 3.0)$ & 2.93 & $1.29-6.64$ & \\
\hline BMI & & & 0.09 \\
\hline Underweight $(<18.5)$ & Ref & & \\
\hline Normal range $(18.5 \leq \mathrm{BMI}<25)$ & 0.38 & $0.09-1.44$ & \\
\hline Overweight/obese (BMI $\geq 25$ ) & 0.88 & $0.21-3.72$ & \\
\hline Menopause & & & 0.28 \\
\hline Premenopausal & Ref & & \\
\hline Postmenopausal & 1.56 & $0.69-3.47$ & \\
\hline $\mathrm{LDH}$ & 1.00 & $1.00-1.00$ & $>0.05$ \\
\hline Subtypes & & & $>0.05$ \\
\hline $\mathrm{ER}^{+}, \mathrm{HER}^{-}$ & Ref & & \\
\hline $\mathrm{ER}^{+}, \mathrm{HER}^{+}$ & 0.28 & $0.05-1.43$ & \\
\hline Triple negative type & 3.31 & $1.01-10.86$ & \\
\hline HER2 type & 0.77 & $0.29-2.08$ & \\
\hline
\end{tabular}

Ref, reference; NLR, neutrophil-to-lymphocyte ratio; BMI, body mass index; LDH, lactate dehydrogenase; ER, estrogen receptor; HER; human epidermal growth factor 2 receptor; CI, confidence interval.

The predictive value of the NLR for survival outcome has been evaluated in several solid malignancies, and a high NLR has been confirmed to be associated with significantly shorter overall survival by meta-analysis (3). Several studies have demonstrated that the pre-treatment NLR predicts survival outcomes for patients with early breast cancer (7-9). In addition, a meta-analysis that included eight studies revealed that an increased NLR was a strong predictor of poor survival (6). However, in five of those studies, the inclusion criteria were inconsistent, and patients with or without metastasis were included. The present study has demonstrated that NLR may be used as a predictive marker for patients not only with pre-treatment status, but also in a metastatic setting.

The present study also analyzed the longitudinal NLR change from pre-treatment to the time of recurrence. The average NLR was increased at the time of recurrence as compared with pre-treatment. Although the difference was not significant, the group with an NLR more than a third quartile higher tended to have shorter OSrec times. To the best of our knowledge, only found three previous studies have analyzed longitudinal NLR changes in colon and hepatocellular carcinoma (12-14); no study has been focused on breast cancer. Those studies focused on the change of NLR from the time of pretreatment to post-treatment, demonstrating that patients with a significantly increased NLR during the treatment term have shorter survival.

The present study also revealed that the inflammatory status upon initial treatment is associated not only with a high recurrence rate, but also with the survival outcome following recurrence. The importance of inflammation for prognosis was also confirmed by another inflammation-based prognostic score, the modified Glasgow prognostic score (mGPS), which was calculated for serum C-reactive protein and albumin, surrogating the degree of the nutrition and inflammatory status in patients with solid malignancies (15-17). Furthermore, the mGPS system was improved by the addition of neutrophil counts (18). Based on this result, it may be postulated that the inflammatory status during the follow-up period after initial treatment exerts a key role in survival following recurrence. Monitoring the NLR during the follow-up period may contribute towards early detection for patients who potentially have a higher risk of recurrence.

$T N$ breast cancer and NLR. The present study demonstrated that TN breast cancer had a significantly higher NLR at the time of recurrence, and the highest degree of NLR change compared with the other subtypes. Previous studies demonstrated that TN breast cancer patients with a high pre-treatment NLR had significantly shorter disease-free and overall survival rates (9). TN breast cancer is aggressive, and demonstrates a high rate of recurrence at an earlier time point following initial treatment (19). That aggressive behavior is promoted by inflammation. For example, the expression of interleukin (IL)-6 and IL-8 inhibits colony formation, cell survival and predicts patient survival (20). A further study also demonstrated that inflammatory cytokines, including tumor necrosis factor- $\alpha$, induce the endothelial-mesenchymal transition in TN breast cancer cells (21). Additionally, a recent clinical trial confirmed that the inflammatory reaction at local tumor sites in TN breast cancer could be indicative of a chemotherapeutic effect, by analyzing the number of tumor-infiltrating lymphocytes $(22,23)$. Taken together, these previously published studies demonstrated that inflammation serves a key role in TN breast cancer progression.

To date, numerous surrogate inflammation markers have been evaluated either in vivo or in vitro for $\mathrm{TN}$ breast cancer, but none have entered common use in a clinical setting due to lack of availability, and their expensiveness. By using NLR as a simple surrogate marker for the degree of inflammation, it is possible to predict survival outcomes, even in patients with metastases. Furthermore, it is possible to determine the current status of inflammation in high-risk patients by monitoring the NLR during the follow-up period. The data associated with longitudinal NLR changes require further refinement in order to confirm the role of inflammation for survival outcome, particularly for the inflammation-based tumor type.

The present study had several limitations. Due to the limited number of patients, the statistical power of the analysis may have been reduced. Secondly, a historical matching group was not used to compare the NLR between recurrent and non-recurrent groups; thus, the difference in the relative NLR change between these two groups was not included in the present study.

In conclusion, it has been demonstrated that an increased NLR predicts survival outcome, even in patients with recurrent breast cancer. In addition, the present study has demonstrated the potential usefulness of NLR as an inflammation marker for $\mathrm{TN}$ breast cancer. 


\section{Acknowledgements}

The authors would like to express their thanks to all patients who participated in this study. This study was presented at the 3rd Advanced Breast Cancer Third International Consensus Conference, 2-4 November 2015, in Lisbon, Portugal.

\section{References}

1. Estimated cancer incidence, mortality and prevalence worldwide in 2012, World Health Organization [Available from: http://globocan.iarc.fr/Pages/fact_sheets_cancer.aspx.]

2. O'Shaughnessy J: Extending survival with chemotherapy in metastatic breast cancer. Oncologist 10 (Suppl 3): S20-S29, 2005

3. Templeton AJ, McNamara MG, Šeruga B, Vera-Badillo FE, Aneja P, Ocana A, Leibowitz-Amit R, Sonpavde G, Knox JJ, Tran B, et al: Prognostic role of neutrophil-to-lymphocyte ratio in solid tumors: A systematic review and meta-analysis. J Natl Cancer Inst 106: dju124, 2014

4. Sharaiha RZ, Halazun KJ, Mirza F, Port JL, Lee PC, Neugut AI, Altorki NK and Abrams JA: Elevated preoperative neutrophil: Lymphocyte ratio as a predictor of postoperative disease recurrence in esophageal cancer. Ann Surg Oncol 18: 3362-3369, 2011.

5. Azab B, Bhatt VR, Phookan J, Murukutla S, Kohn N, Terjanian T and Widmann WD: Usefulness of the neutrophil-to-lymphocyte ratio in predicting short- and long-term mortality in breast cancer patients. Ann Surg Oncol 19: 217-224, 2012.

6. Chen J, Deng Q, Pan Y, He B, Ying H, Sun H, Liu X and Wang S: Prognostic value of neutrophil-to-lymphocyte ratio in breast cancer. FEBS Open Bio 5: 502-507, 2015.

7. Noh H, Eomm M and Han A: Usefulness of pretreatment neutrophil to lymphocyte ratio in predicting disease-specific survival in breast cancer patients. J Breast Cancer 16: 55-59, 2013.

8. Azab B, Shah N, Radbel J, Tan P, Bhatt V, Vonfrolio S, Habeshy A, Picon A and Bloom S: Pretreatment neutrophil/lymphocyte ratio is superior to platelet/lymphocy te ratio as a predictor of long-term mortality in breast cancer patients. Med Oncol 30: 432, 2013.

9. Pistelli M, De Lisa M, Ballatore Z, Caramanti M, Pagliacci A, Battelli N, Ridolfi F, Santoni M, Maccaroni E, Bracci R, et al: Pre-treatment neutrophil to lymphocyte ratio may be a useful tool in predicting survival in early triple negative breast cancer patients. BMC Cancer 15: 195, 2015

10. Edge SB BD, Compton CC, Fritz AG, Greene FL and Trotti A: Ajcc cancer staging manual. 7th ed. Springer, New York, 2010.

11. Hortobagyi GN: Treatment of breast cancer. N Engl J Med 339: 974-984, 1998.
12. Dan J, Zhang Y, Peng Z, Huang J, Gao H, Xu L and Chen M: Postoperative neutrophil-to-lymphocyte ratio change predicts survival of patients with small hepatocellular carcinoma undergoing radiofrequency ablation. PloS One 8: e58184, 2013.

13. Guthrie GJ, Roxburgh CS, Farhan-Alanie OM, Horgan PG and McMillan DC: Comparison of the prognostic value of longitudinal measurements of systemic inflammation in patients undergoing curative resection of colorectal cancer. Br J Cancer 109: 24-28, 2013.

14. Peng W, Li C, Wen TF, Yan LN, Li B, Wang WT, Yang JY and Xu MQ: Neutrophil to lymphocyte ratio changes predict small hepatocellular carcinoma survival. J Surg Res 192: 402-408, 2014.

15. Li MX, Bi XY, Li ZY, Huang Z, Han Y, Zhou JG, Zhao JJ, Zhang YF, Zhao $\mathrm{H}$ and Cai JQ: Prognostic role of glasgow prognostic score in patients with hepatocellular carcinoma: A systematic review and meta-analysis. Medicine (Baltimore) 94: e2133, 2015.

16. Roxburgh CS, Salmond JM, Horgan PG, Oien KA and McMillan DC: Comparison of the prognostic value of inflammation-based pathologic and biochemical criteria in patients undergoing potentially curative resection for colorectal cancer. Ann Surg 249: 788-793, 2009.

17. Sheng T, Wang B, Wang SY, Deng B, Qu L, Qi XS, Wang XL, Deng GL and Sun X: The relationship between serum interleukin- 6 and the recurrence of hepatitis b virus related hepatocellular carcinoma after curative resection. Medicine (Baltimore) 94: e941, 2015.

18. Proctor MJ, Horgan PG, Talwar D, Fletcher CD, Morrison DS and McMillan DC: Optimization of the systemic inflammation-based glasgow prognostic score: A glasgow inflammation outcome study. Cancer 119: 2325-2332, 2013.

19. Foulkes WD, Smith IE and Reis-Filho JS: Triple-negative breast cancer. N Engl J Med 363: 1938-1948, 2010.

20. Hartman ZC, Poage GM, den Hollander P, Tsimelzon A, Hill J, Panupinthu N, Zhang Y, Mazumdar A, Hilsenbeck SG, Mills GB and Brown PH: Growth of triple-negative breast cancer cells relies upon coordinate autocrine expression of the proinflammatory cytokines il-6 and il-8. Cancer Res 73: 3470-3480, 2013.

21. Qiao Y, Shiue CN, Zhu J, Zhuang T, Jonsson P, Wright AP, Zhao C and Dahlman-Wright K: Ap-1-mediated chromatin looping regulates ZEB2 transcription: New insights into tnfo-induced epithelial-mesenchymal transition in triple-negative breast cancer. Oncotarget 6: 7804-7814, 2015.

22. Adams S, Gray RJ, Demaria S, Goldstein L, Perez EA, Shulman LN, Martino S, Wang M, Jones VE, Saphner TJ, et al: Prognostic value of tumor-infiltrating lymphocytes in triple-negative breast cancers from two phase III randomized adjuvant breast cancer trials: ECOG 2197 and ECOG 1199. J Clin Oncol 32: 2959-2966, 2014.

23. Matsumoto H, Koo SL, Dent R, Tan PH and Iqbal J: Role of inflammatory infiltrates in triple negative breast cancer. J Clin Pathol 68: 506-510, 2015. 